PIXTE

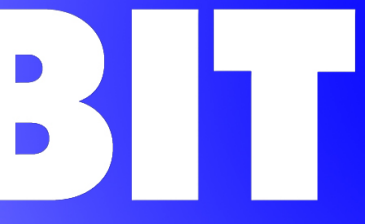

№ 57 ENERO 2020

CUATRIMESTRAL

e-ISSN:2171-7966

ISSN:1133-8482

\title{
Revista de Medios y Educación
}

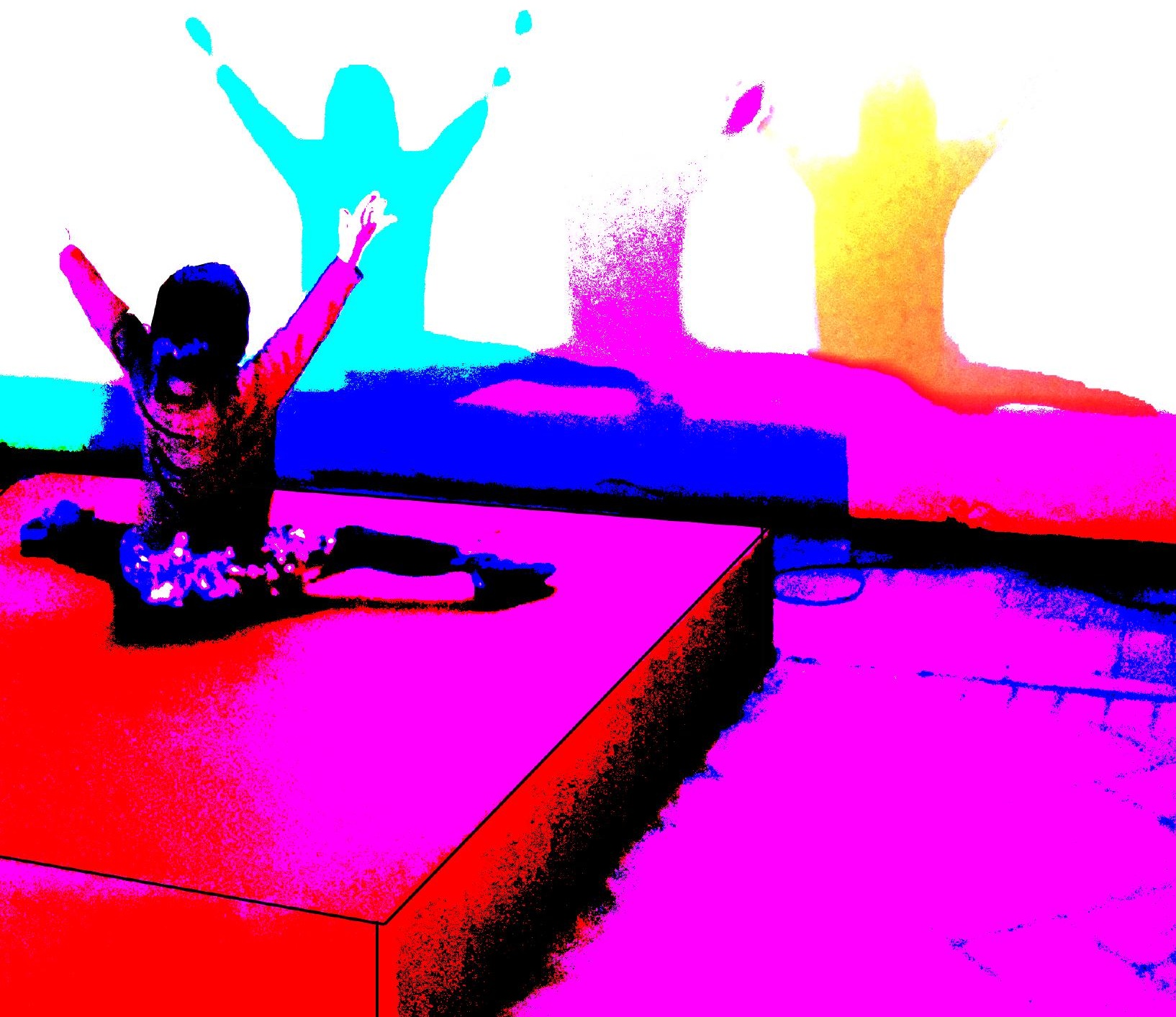




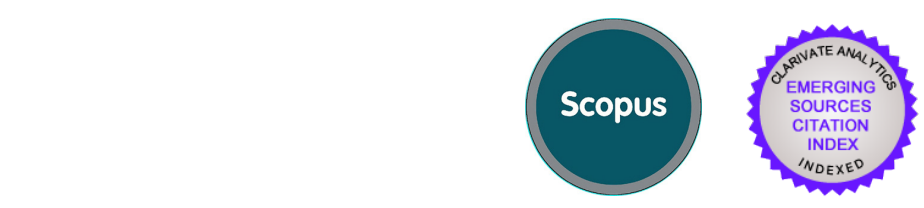

\section{PIXEL-BIT}

\section{REVISTA DE MEDIOS Y EDUCACIÓN}

No 57 - ENERO- 2020

https://recyt.fecyt.es/index.php/pixel/index 


\section{EQUIPO EDITORIAL (EDITORIAL BOARD)}

EDITOR JEFE (EDITOR IN CHIEF)

Dr. Julio Cabero Almenara, Departamento de Didáctica y Organización Educativa, Facultad de CC de la Educación, Universidad de Sevilla (España).

EDITOR ADJUNTO (ASSISTANT EDITOR)

Dr. Óscar M. Gallego Pérez, Secretariado de Recursos Audiovisuales y NN.TT., Universidad de Sevilla (España)

EDITOR EJECUTIVO/SECRETARIO GENERAL EDITORIAL (EXECUTIVE EDITOR)

Dr. Juan Jesús Gutiérrez Castillo, Departamento de Didáctica y Organización Educativa. Facultad de CC de la Educación, Universidad de Sevilla (España).

\section{CONSEJO DE REDACCIÓN}

\section{EDITOR}

Dr. Julio Cabero Almenara. Universidad de Sevilla (Espańa)

\section{EDITOR ASISTENTE}

Dr. Óscar M. Gallego Pérez. Universidad de Sevilla (España)

\section{SECRETARIO}

Dr. Juan Jesús Gutiérrez Castillo. Universidad de Sevilla (Espańa)

\section{VOCALES}

Dra. María Puig Gutiérrez, Universidad de Sevilla. (Espańa)

Dra. Sandra Martínez Pérez, Universidad de Barcelona (Espańa)

Dr. Selín Carrasco, Universidad de La Punta (Argentina)

Dr. Jackson Collares, Universidades Federal do Amazonas (Brasil)

Dra. Kitty Gaona, Universidad Autónoma de Asunción (Paraguay)

Dra. Elvira Esther Navas, Universidad Metropolitana de Venezuela (Venezuela)

Dr. Angel Puentes Puente, Pontificia Universidad Católica Madre y Maestra. Santo Domingo (República Dominicana)

Dr. Fabrizio Manuel Sirignano, Università degli Studi Suor Orsola Benincasa (Italia)

\section{CONSEJO TÉCNICO}

Edición, maquetación: Manuel Serrano Hidalgo, Universidad de Sevilla (España)

Diseño de portada: Lucía Terrones García, S.A.V, Universidad de Sevilla (España)

Revisor/corrector de textos en inglés: Rubicelia Valencia Ortiz, MacMillan Education (México)

Revisores metodológicos: evaluadores asignados a cada artículo

Responsable de redes sociales: Manuel Serrano Hidalgo, Universidad de Sevilla (Espańa)

Bases de datos: Bárbara Fernández Robles, Universidad de Sevilla (Espańa)

Administración: Leticia Pinto Correa, S.A.V, Universidad de Sevilla (España)

\section{CONSEJO CIENTÍFICO}

Jordi Adell Segura, Universidad Jaume I Castellón (España)

Ignacio Aguaded Gómez, Universidad de Huelva (España)

María Victoria Aguiar Perera, Universidad de Las Palmas de Gran Canaria (España)

Olga María Alegre de la Rosa, Universidad de la Laguna Tenerife (Espańa)

Manuel Área Moreira, Universidad de la Laguna Tenerife (Espańa)

Patricia Ávila Muñoz, Instituto Latinoamericano de Comunicación Educativa (México)

Antonio Bartolomé Pina, Universidad de Barcelona (España)

Angel Manuel Bautista Valencia, Universidad Central de Panamá (Panamá)

Jos Beishuizen, Vrije Universiteit Amsterdam (Holanda)

Florentino Blázquez Entonado, Universidad de Extremadura (Espańa)

Silvana Calaprice, Università degli studi di Bari (Italia)

Selín Carrasco, Universidad de La Punta (Argentina) 
Raimundo Carrasco Soto, Universidad de Durango (México)

Rafael Castañeda Barrena, Universidad de Sevilla (España)

Zulma Cataldi, Universidad de Buenos Aires (Argentina)

Manuel Cebrián de la Serna, Universidad de Málaga (Espańa)

Luciano Cecconi, Università degli Studi di Modena (Italia)

Jean-François Cerisier, Université de Poitiers, Francia

Jordi Lluís Coiduras Rodríguez, Universidad de Lleida (España)

Jackson Collares, Universidades Federal do Amazonas (Brasil)

Enricomaria Corbi, Università degli Studi Suor Orsola Benincasa (Italia)

Marialaura Cunzio, Università degli Studi Suor Orsola Benincasa (Italia)

Brigitte Denis, Université de Liège (Bélgica)

Floriana Falcinelli, Università degli Studi di Perugia (Italia)

Maria Cecilia Fonseca Sardi, Universidad Metropolitana de Venezuela (Venezuela)

Maribel Santos Miranda Pinto, Universidade do Minho (Portugal)

Kitty Gaona, Universidad Autónoma de Asunción (Paraguay)

María-Jesús Gallego-Arrufat, Universidad de Granada (España)

Fernando Gamboa Rodríguez, Universidad Nacional Autónoma de México

Lorenzo García Aretio, UNED (Espańa)

Ana García-Valcarcel Muńoz-Repiso, Universidad de Salamanca (España)

Antonio Bautista García-Vera, Universidad Complutense de Madrid (España)

José Manuel Gómez y Méndez, Universidad de Sevilla (España)

Mercedes González Sanmamed, Universidad de La Coruña (España)

Manuel González-Sicilia Llamas, Universidad Católica San Antonio-Murcia (España)

Ángel Pio González Soto, Universidad Rovira i Virgili, Tarragona (España)

António José Meneses Osório, Universidade do Minho (Portugal)

Carol Halal Orfali, Universidad Tecnológica de Chile INACAP (Chile)

Mauricio Hernández Ramírez, Universidad Autónoma de Tamaulipas (México)

Ana Landeta Etxeberría, Universidad a Distancia de Madrid (UDIMA)

Linda Lavelle, Plymouth Institute of Education (Inglaterra)

Fernando Leal Ríos, Universidad Autónoma de Tamaulipas (México)

Paul Lefrere, Cca (UK)

Carlos Marcelo García, Universidad de Sevilla (España)

Francois Marchessou, Universidad de Poittiers, París (Francia)

Francesca Marone, Università degli Studi di Napoli Federico II (Italia)

Francisco Martínez Sánchez, Universidad de Murcia (España)

Ivory de Lourdes Mogollón de Lugo, Universidad Central de Venezuela (Venezuela)

Angela Muschitiello, Università degli studi di Bari (Italia)

Margherita Musello, Università degli Studi Suor Orsola Benincasa (Italia)

Elvira Esther Navas, Universidad Metropolitana de Venezuela (Venezuela)

Trinidad Núńez Domínguez, Universidad de Sevilla (España)

James O’Higgins, de la Universidad de Dublín (UK)

José Antonio Ortega Carrillo, Universidad de Granada (Espańa)

Gabriela Padilla, Universidad Autónoma de Tumalipas (México)

Ramón Pérez Pérez, Universidad de Oviedo (España)

Angel Puentes Puente, Pontificia Universidad Católica Madre y Maestra. Santo Domingo (República Dominicana)

Julio Manuel Barroso Osuna, Universidad de Sevilla (España)

Rosalía Romero Tena. Universidad de Sevilla (España)

Hommy Rosario, Universidad de Carabobo (Venezuela)

Pier Giuseppe Rossi, Università di Macerata (Italia)

Jesús Salinas Ibáńez, Universidad Islas Baleares (España)

Yamile Sandoval Romero, Universidad de Santiago de Cali (Colombia)

Albert Sangrá Morer, Universidad Oberta de Catalunya (Espańa)

Ángel Sanmartín Alonso, Universidad de Valencia (España)

Horacio Santángelo, Universidad Tecnológica Nacional (Argentina)

Francisco Solá Cabrera, Universidad de Sevilla (España)

Jan Frick, Stavanger University (Noruega)

Karl Steffens, Universidad de Colonia (Alemania)

Seppo Tella, Helsinki University (Finlandia)

Hanne Wacher Kjaergaard, Aarhus University (Dinamarca) 


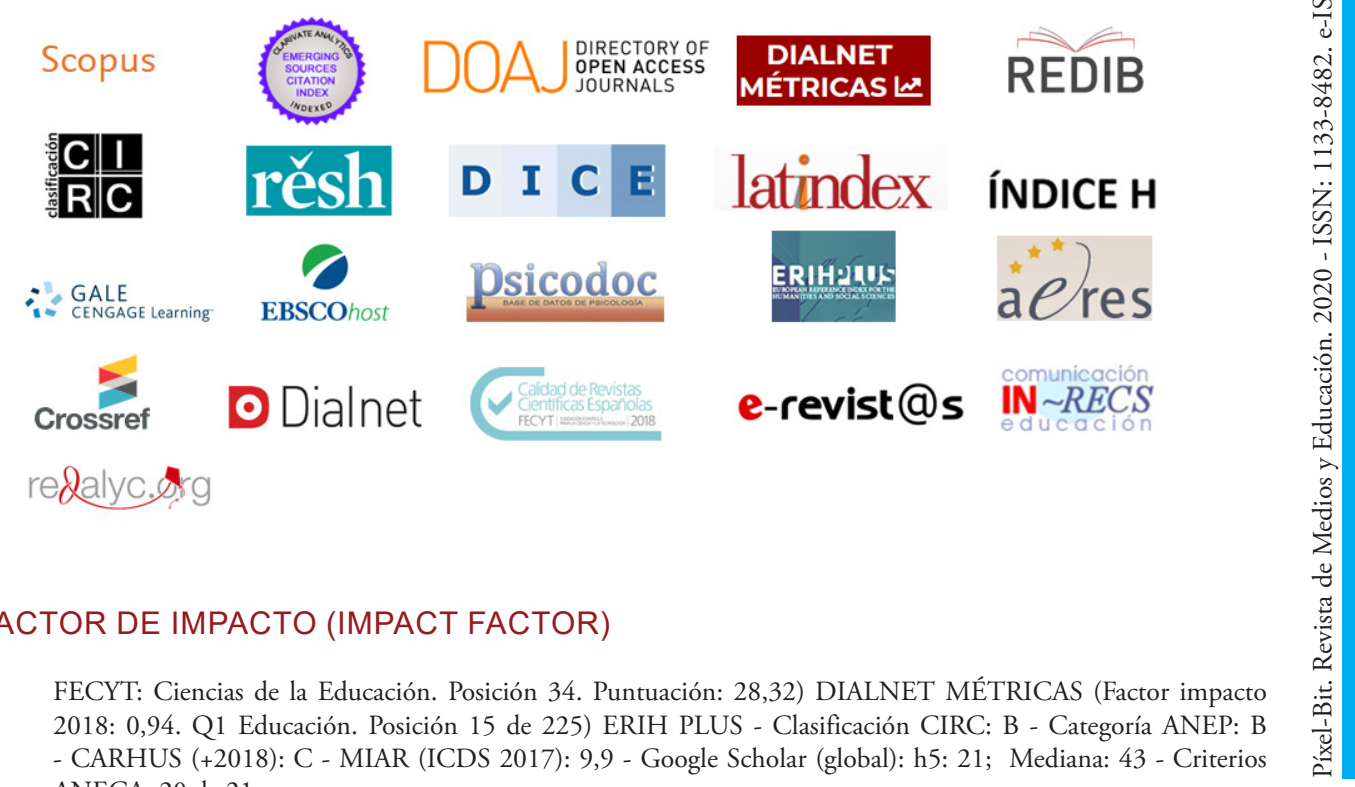

Píxel-Bit, Revista de Medios y Educación está indexada entre otras bases en: SCOPUS, Fecyt, Iresie, ISOC (CSIC/ CINDOC), DICE, MIAR, IN-RECS, RESH, Ulrich's Periodicals, Catálogo Latindex, Biné-EDUSOL, Dialnet, Redinet, OEI, DOCE, Scribd, Redalyc, Red Iberoamericana de Revistas de Comunicación y Cultura, Gage Cengage Learning, Centro de Documentación del Observatorio de la Infancia en Andalucía. Además de estar presente en portales especializados, Buscadores Científicos y Catálogos de Bibliotecas de reconocido prestigio, y pendiente de evaluación en otras bases de datos.

\section{EDITA (PUBLISHED BY)}

Grupo de Investigación Didáctica (HUM-390). Universidad de Sevilla (Espańa). Facultad de Ciencias de la Educación. Departamento de Didáctica y Organización Educativa. C/ Pirotecnia s/n, 41013 Sevilla.

Dirección de correo electrónico: revistapixelbit@us.es . URL: https://recyt.fecyt.es/index.php/pixel/index ISSN: 1133-8482; e-ISSN: 2171-7966; Depósito Legal: SE-1725-02

Formato de la revista: $16,5 \times 23,0 \mathrm{~cm}$

Los recursos incluidos en Píxel Bit están sujetos a una licencia Creative Commons Attribution-NonCommercialShareAlike 3.0 Unported (Reconocimiento-NoComercial-CompartirIgual)(CC BY-NC-SA 3.0), en consecuencia, las acciones, productos y utilidades derivadas de su utilización no podrán generar ningún tipo de lucro y la obra generada sólo podrá distribuirse bajo esta misma licencia. En las obras derivadas deberá, asimismo, hacerse referencia expresa a la fuente y al autor del recurso utilizado.

(C2020 Píxel-Bit. No está permitida la reproducción total o parcial por ningún medio de la versión impresa de la Revista Píxel- Bit. 
1.- Construtivist and collaborative methodology mediated by ICT in Higher Education // Constructivismo y metodología colaborativa mediada por TIC en educación superior usando webquest (Bilingue)

María Carmen Corujo Vélez, María Teresa Gómez del Castillo, Alma Elizabeth Merla-González

2.- Big Data Irruption in Education // Irrupción del Big Data en la Educación (Bilingue)

Pablo Daniel Franco Caballero, Antonio Matas Terrón, Juan José Leiva Olivencia

3.- Codiseño de un chatbot para facilitar procedimientos administrativos a población migrada // Codesign of a chatbot to facilitate administrative procedures for migrants

Begoña Gros Salvat, Anna Escofet Roig, Montserrat Payá Sánchez

4.-Metodologías y Tecnologías para enseñar STEM en Educación Primaria: análisis de necesidades // Methodologies \& Technologies to teach STEM in Primary Education: needs analysis

Javier Arabit García, Ma Paz Prendes Espinosa

5.- Conocimiento de los estudiantes universitarios sobre herramientas antiplagio y medidas preventivas // University students knowledge on anti-plagiarism tolls and preventive measures

Violeta Cebrián-Robles, Manuela Raposo-Rivas, Francisco José Ruiz-Rey

6.- Perfiles de acceso y apropiación de TIC en ingresantes universitarios. Estudio comparativo en dos universidades públicas argentinas // Profiles of access and appropriation of ICT in freshmen students. Comparative study in two Argentine public universities.

María Luisa Bossolasco, Analía Claudia Chiecher, Daniel Andrés Dos Santos

7.- Validación de escala MPPUS-A sobre el uso problemático del smartphone // Validation of scale MPPUS-A on the problematic use of the smartphone

Andrés García-Umaña, Évelyn Córdoba Pillajo

8.- Desarrollo del oído absoluto a través de aplicaciones móviles // Development of the absolut pitch through mobile applications

Rosabel Roig-Vila, Facundo San Blas, Àngela Buforn

9.- Construcción del concepto fanbullying: Revisión crítica del acoso en redes sociales // Building the fanbullying concept: Critical review of social media harassment

Arantxa Vizcaíno-Verdú, Paloma Contreras-Pulido, María Dolores Guzmán-Franco

10.- Microblogging educativo en el aprendizaje de idiomas: una revisión sistematizada de las investigaciones publicadas entre 2010 y 2018 // Educational Microblogging for Language Learning: A Systematised Review of Research Publications between 2010 and 2018

Marta Rosón Jiménez, Daniel Losada Iglesias 


\title{
Desarrollo del oído absoluto a través de aplicaciones móviles
}

\section{Development of the absolut pitch through mobile applications}

\author{
Dra. Rosabel Roig-Vila ${ }^{1}$ rosabel.roig@ua.es \\ D. Facundo San Blas Arenes ${ }^{2}$ facun_band@hotmail.com \\ Dra. Àngela Buforn Lloret ${ }^{3}$ angela.buforn@ua.es
}

\begin{abstract}
${ }^{1}$ Universidad de Alicante. Facultad de Educación. Departamento de Didáctica General y Didáctica específicas. Campus Sant Vicent del Raspeig. Alicante (Espańa).

${ }^{2}$ Escola de Música Ateneu La Vila Joiosa. Villajoyosa. Alicante (España)

${ }^{3}$ Universidad de Alicante. Facultad de Educación. Departamento de Innovación y Formación Didáctica. Campus

Sant Vicent del Raspeig. Alicante (Espańa).
\end{abstract}

\section{RESUMEN}

En este estudio se analiza si influye el uso de aplicaciones móviles (apps) basadas en el método musical "Mago Diapasón" en niños de dos años de edad con respecto al desarrollo del oído absoluto. Los participantes del estudio han sido 19 niños/ as escolarizados en dos centros infantiles. Diez de ellos pertenecían al grupo experimental y los nueve restantes al grupo control. Los participantes del grupo control asistían a clases semanales de esta metodología, y los del grupo experimental, además de asistir a las clases, estuvieron manipulando las apps durante seis meses con la ayuda de los padres. Con el fin de analizar el proceso de aprendizaje, se pasaron un par de cuestionarios (pre-test y posttest) que consistían en la realización de un dictado melódico (sin ritmo). Los resultados muestran que los participantes que han utilizado las apps (grupo experimental), asistiendo, además, a las clases de esta metodología, han empezado a desarrollar el oído absoluto de manera más significativa que los participantes que solamente han asistido a las clases semanales de música. Este resultado sugiere que las apps pueden contribuir al desarrollo del oído absoluto desde tempranas edades.

\section{PALABRAS CLAVE}

Educación musical; educación de la primera infancia; programa informático didáctico; enseñanza multimedia; método de enseñanza.

\section{ABSTRACT}

In this study, the influence of the use of mobile applications (apps) based on the musical method "Mago Diapasón" in two-year-old children with respect to the development of the absolute ear is analyzed. The participants of the study were 19 children enrolled in two childhood education schools. Ten of them belonged to the experimental group and the remaining nine belonged to the control group. Participants of the control group attended weekly classes of this methodology, and those of the experimental group, a part of attending the classes, manipulated the applications for six months with the help of their parents. In order to analyse the learning process, we passed a couple of questionnaires (pre-test and post-test) that consisted of a melodic dictation (without rhythm). The results showed that the students who have used the applications (experimental group), a part of attend the classes of this methodology, have begun to develop the absolute pitch in a more meaningful way than the participants who only attended the weekly music classes. This result suggests that applications can contribu-te to the development of absolute judgment from early ages.used by social organizations.

\section{KEYWORDS}

Musical education; early childhood education; didactic computer program; multimedia teaching; teaching method. 


\section{1.- Introducción}

\subsection{Oído absoluto en el contexto social}

Cuando hablamos de oído absoluto (OA) u oído perfecto nos referimos a la capacidad de identificar la frecuencia de un estímulo auditivo aislado, sin la ayuda de un estímulo auditivo referencial (Moulton, 2014). Así pues, se podría definir, básicamente, como la capacidad de reconocer los sonidos. Un ejemplo de oído absoluto lo podemos observar en grandes genios de la música como, por ejemplo, Mozart, Bach y Tchaikovski, entre otros. El hecho de entrenar y desarrollar el oído desde edades tempranas es recomendable para que los niños aprendan a relacionar la discriminación de las alturas en cuanto a sonido se refiere. De hecho, y coincidiendo con diferentes investigadores, para desarrollar el oído absoluto es importante introducirse en el estudio de la música antes de los 6 años (Baharloo, Johnston, Gitschie, \& Freimer, 1998; Baharloo, Risch, Gitschier, \& Freimer, 2000), ya que así las probabilidades de su desarrollo se verán más ampliadas.

Respecto a la posesión del OA, encontramos diferentes opiniones. Radocy y Boyle (1988) piensan que la posesión de $\mathrm{OA}$ es algo sorprendente para alguien que no lo posee y puede llegar a ser molesto para las personas con esta cualidad. Así, el desarrollo del OA está totalmente ligado a los beneficios que se obtienen para el estudio de un instrumento musical o, desde otra perspectiva, ligado a la diversidad dentro de la población normal, como es, por ejemplo, el autismo (Dohn, Garza-Villarreal, Heaton \& Vuust, 2012; Heaton, 2009). Según Barkowsky (1987), Stumpf fue la primera persona que estudió científicamente el OA en torno a 1883. Este autor defendía que el OA va unido a lo que se considera aptitud musical y su posesión permite disfrutar plenamente de las obras musicales. Según Zielinska y Miklaszewski (1992), el OA ayuda a memorizar muy rápidamente las melodías. Es por ello que los sujetos con OA son mejores que los que tienen oído relativo, tanto en melodías tonales como modales. Además, las personas con OA tienen más facilidad para recordar melodías a largo plazo, ya que destacan en la retención de la tonalidad y en el almacenamiento de la información tonal.

Por otro lado, el OA supone una ayuda para el desarrollo del oído armónico y la percepción de modulaciones. Estos parámetros nos permiten una percepción más analítica de la música y, en consecuencia, la posibilidad de perfeccionar su conocimiento (Shuter-Dyson \& Gabriel, 1981). También Miyazaki (1994, 1995) argumenta que el OA permite la audición interior ante la lectura de un texto musical, la 
reproducción vocal de los sonidos que lo componen con elevada precisión, el favorecimiento de la lectura a primera vista y la realización del dictado musical. Además, es de gran utilidad para directores de coro o de orquesta en lo relativo a la afinación. Por su parte, Nering (1991) señala el OA como beneficioso para "tocar a oído, la improvisación, la seguridad vocal, las destrezas tonales, la memorización y la apreciación musical”.

Respecto a cómo educar el oído absoluto y cuándo empezar a trabajarlo, se han encontrado diferentes investigaciones. Baharloo et al. $(1998,2000)$ defienden que el entrenamiento temprano en la música es un importante factor ambiental que contribuye al desarrollo de OA. Además, en diversos estudios encontraron que los hermanos pequeños de los niños que estudian música en casa tienen más probabilidades de estar expuestos a la formación musical temprana y, por lo tanto, a tener una mayor probabilidad de desarrollar OA (Baharloo et al., 2000). Asimismo, según Vanzella y Schellenberg (2010), las lecciones de piano u otros instrumentos de tono fijo parecen mejorar las habilidades del OA y extender el período sensible para la exposición a la música con el fin de desarrollar un OA más preciso. Además, otras de las habilidades que poseen las personas con OA son la rapidez y precisión de acierto en el reconocimiento de los sonidos. En el trabajo de Miyazaki (1988), los resultados diferenciaron claramente a los poseedores de los no poseedores de OA a través de la precisión y velocidad de respuesta. Aquellos sujetos que tenían OA podían clasificar los tonos de forma consistente mediante el uso de las cualidades musicales de los tonos (tono cromático). Sin embargo, no respondieron de manera uniforme a todos los estímulos; respondieron con mayor precisión y rapidez a algunos tonos musicalmente importantes en un modo Do-mayor (Do, Mi, o Sol). Por otro lado, aquellos que no tenían OA mostraron patrones de respuesta casi aleatorios.

En el trabajo de Miyazaki (1988), los resultados diferenciaron claramente a los poseedores de los no poseedores de OA a través de la precisión y velocidad de respuesta. Aquellos sujetos que tenían OA podían clasificar los tonos de forma consistente mediante el uso de las cualidades musicales de los tonos (tono croma). Sin embargo, no respondieron de manera uniforme a todos los estímulos; respondieron con mayor precisión y rapidez a los tonos relacionados con el modo Do-mayor (Do, Mi, o Sol). Por otro lado, aquellos que no tenían OA mostraron patrones de respuesta casi aleatorios. En este contexto musical, el efecto croma en los poseedores de OA, afecta positivamente a la organización memotécnica 
de las características musicales que poseen los tonos musicales, a diferencia de los no poseedores de OA. Estos, con la ausencia de contexto musical, tienen mucha más dificultad para reconocer dichos tonos. Además, previamente al estudio de Miyazaki (1988), se creía que el OA era una cualidad rara. En nuestro estudio se ha observado la correlación entre la posesión de OA y el entrenamiento musical temprano.

\subsection{TIC y Música}

De acuerdo con Morel (2016), las Tecnologías de la Información y la Comunicación (TIC) cobran cada día mayor importancia en todos los ámbitos de la vida cotidiana, tanto sociales (Reinking \& Martin, 2018), como culturales y educativos (García-Umaña \& Tirado-Morueta, 2018), entre otros. En particular, en el ámbito educativo, las TIC son una herramienta actual que amplían y facilitan el apoyo didáctico, actuando como un recurso educativo que fomenta una metodología de enseñanza más abierta hacia el autoaprendizaje (Creer, 2018). Teniendo en cuenta que no todos los alumnos aprenden de la misma manera, el uso de las TIC favorece que el alumnado relacione la materia con el mundo real y pueda participar de forma activa en su propio aprendizaje. De este modo, el alumnado juega e interactúa con el objeto de estudio, aumenta su motivación y mantiene el carácter de reto que implica la educación, donde, por semejanza con la literatura, explora la realidad que le rodea, imagina otros mundos y entiende otras perspectivas (Arlandis \& Reyes-Torres, 2018).

De acuerdo con Sánchez (2003), integrar curricularmente las TIC implica tenerlas en cuenta en la metodología que facilita aprender al aprendiz, tanto en el aula, como fuera de ella (Froehlich, 2018). Según Claro (2010), la tecnología mejoraría el rendimiento escolar de los estudiantes al implicar cambios en las estrategias de enseñanza y aprendizaje. La mayor capacidad de conexión, la convergencia de pantallas entre televisión, ordenadores y dispositivos móviles, y el desarrollo de tecnologías audiovisuales alternativas, tales como la realidad aumentada (Cabero-Almenara \& Roig-Vila, 2019; Roig-Vila, Lorenzo-Lledó \& Mengual-Andrés, 2019), hologramas, videojuegos (Marín-Díaz, Morales-Díaz \& Reche-Urbano, 2019), etc., enmarcan el entorno tecnológico en el que se educan los niños y niñas de hoy, resultando completamente natural para los mismos (Martino \& Frida, 2014), pero siempre teniendo en cuenta los posibles riesgos (García-Umaña \& Tirado-Morueta, 2018).

En este estudio nos centraremos en la educación musical y el uso de las TIC. Con respecto a la literatura sobre este tándem, encontramos algunas investigaciones, como la de Lánchez (2014) que examina el 
aprendizaje de la lectoescritura musical mediante el uso de las TIC de una manera lúdica. Por otro lado, Hincapié y Graciano (2016) tienen como propósito caracterizar el desarrollo de los procesos de lectoescritura, la música, las TIC y las artes visuales en la utilización de estrategias didácticas, basadas en el uso de los cuentos infantiles, rondas, expresiones artísticas y audiovisuales, entre otros. Cabe destacar también trabajos como el de Llargués, Niño, Rodríguez y Segura (2015) que estudian el uso que se les da a las TIC en las clases de música en la educación secundaria, el cual consiste en la búsqueda enciclopédica de información, u otras finalidades, que no involucran al ordenador como un medio de producción musical.

En este estudio nos centramos en cómo el uso de las TIC, en concreto el uso de apps, pueden ayudar a desarrollar el oído absoluto siguiendo la metodología del Mago Diapasón que explicaremos a continuación.

\subsection{Método Mago Diapasón}

El Mago Diapasón es una metodología diseńada para niños de entre 6 meses y 8 años, cuyos objetivos principales son la enseñanza del lenguaje musical y el desarrollo del oído absoluto. En dicha metodología los niños se sumergen en un cuento diseñado de manera estratégica para que el profesor sea capaz de captar la atención a edades tempranas y realizar un aprendizaje real y significativo de manera amena y divertida. Para ello, la metodología cuenta con recursos y herramientas que atraen el interés del niño y por su variedad de contenido, tanto visual, auditivo como gestual, mantienen la focalización durante toda la clase. La Figura 1 muestra los principales protagonistas del cuento: el Mago Diapasón, Esther, Álex, Pablo y Sara (de izquierda a derecha). A través del cuento, se aprende la teoría de la música y se les enseñan las notas musicales La, Do, Mi y Sol, mediante la escala de fononímia de Kódaly; además, estas notas se relacionan con las emociones básicas. Cada una de las notas tiene su propia canción y se van introduciendo de manera progresiva. En primer lugar, se aprende la nota La, que es la nota que canta la varita mágica del Mago Diapasón (nota alegre). Además, ésta coincide con el sonido de un diapasón normal, por lo que nos sirve para medir el valor absoluto de los tonos y para poder afinar a partir de esta nota (Pastor, 2008). En segundo lugar, aparece la nota Do (nota enfadada porque quiere ser importante). A medida que la historia avanza, se les enseńa la nota Mi (nota que llora) y, por último, aparece la nota Sol que llega para ayudar a todas las notas a que sean buenas y se porten bien. De esta manera se descubre el arpegio de Do mayor y la nota Do ya es una nota importante. 


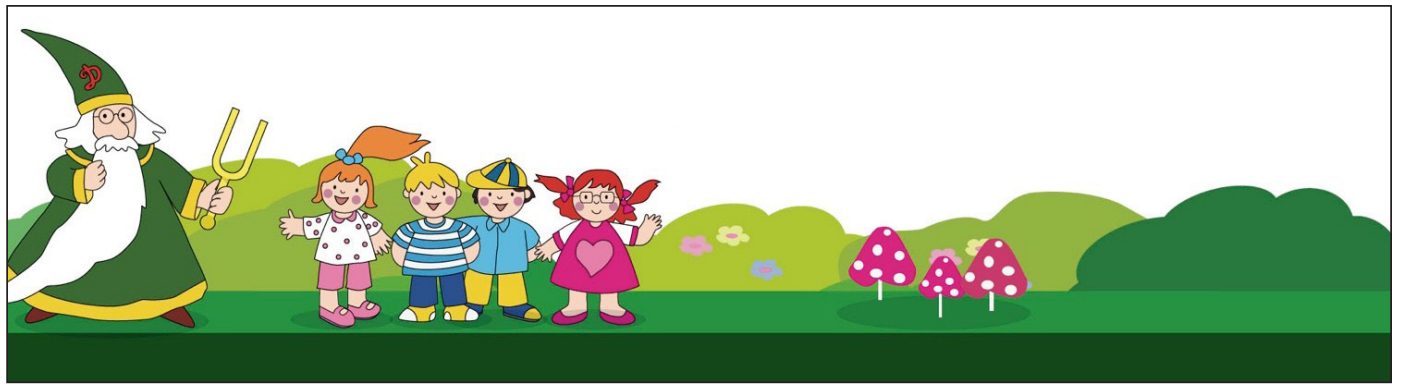

Figura 1. Protagonistas del cuento del Mago Diapasón

Mediante las canciones que se emplean, los niños sienten la necesidad de introducirse dentro de la música, bien sea mediante gestos o palmas. Además, en estas canciones también se cantan de manera lateral las notas, con la finalidad de que se vaya reconociendo su afinación. Estas canciones son simples y están compuestas especialmente para ser agradables y fáciles de aprender.

Este método puede seguirse de forma tradicional o utilizando la aplicación móvil diseñada para tal fin. Los usuarios, en este caso padres, pueden acceder a este material mediante la plataforma online que se les habilita. La app contiene cuentos donde las notas se presentan con juegos y herramientas divertidas para reconocer los sonidos (las notas). Los padres, así, ayudan a los niños a aprender el lenguaje musical y a desarrollar el oído absoluto sin conocimientos musicales por su parte.

La app, en este caso, puede ser un aliado para el proceso de enseñanza/aprendizaje, ya que el sistema Android se puede manipular de manera táctil y su gestión es muy simple para los niños. En concreto, Mago Diapasón dispone de cinco aplicaciones formadas por un cuento de cinco páginas interactivas y dos juegos. Cada una de estas aplicaciones presenta diferente nivel de dificultad, es decir, cada aplicación tiene un nivel y en cada uno de estos niveles se trabajan progresivamente determinadas notas:

- Nivel 2. El patito. Notas LA y DO.

- Nivel 3. La clave de sol. Notas LA, DO y MI.

- Nivel 4. Ron y turrón. Notas LA, DO y MI.

- Nivel 5. La clave de fa. Notas LA, DO, MI y SOL.

Así por ejemplo, la aplicación "el Pentagrama" (Figura 2) consta de 5 pantallas y en cada pantalla se explican, de manera breve y en forma de cuento, algunas definiciones de los conceptos teóricos más 
esenciales para introducirnos en el mundo de la música. Además, cada pantalla dispone de varios iconos que se mueven o se realzan para que, cuando presiones sobre ellos, suene una nota (en este nivel solo aparecen las notas La y Do). De este modo, el nińo escucha estas notas y aprende a cantarlas. Una vez transcurridas las 5 páginas, la aplicación nos lleva a dos juegos: uno en el que suenan notas y hay que acertarlas, y otro de emparejar cartas según los sonidos. Cuando se acierta se obtiene un diapasón como premio y si se falla se pierde un diapasón.

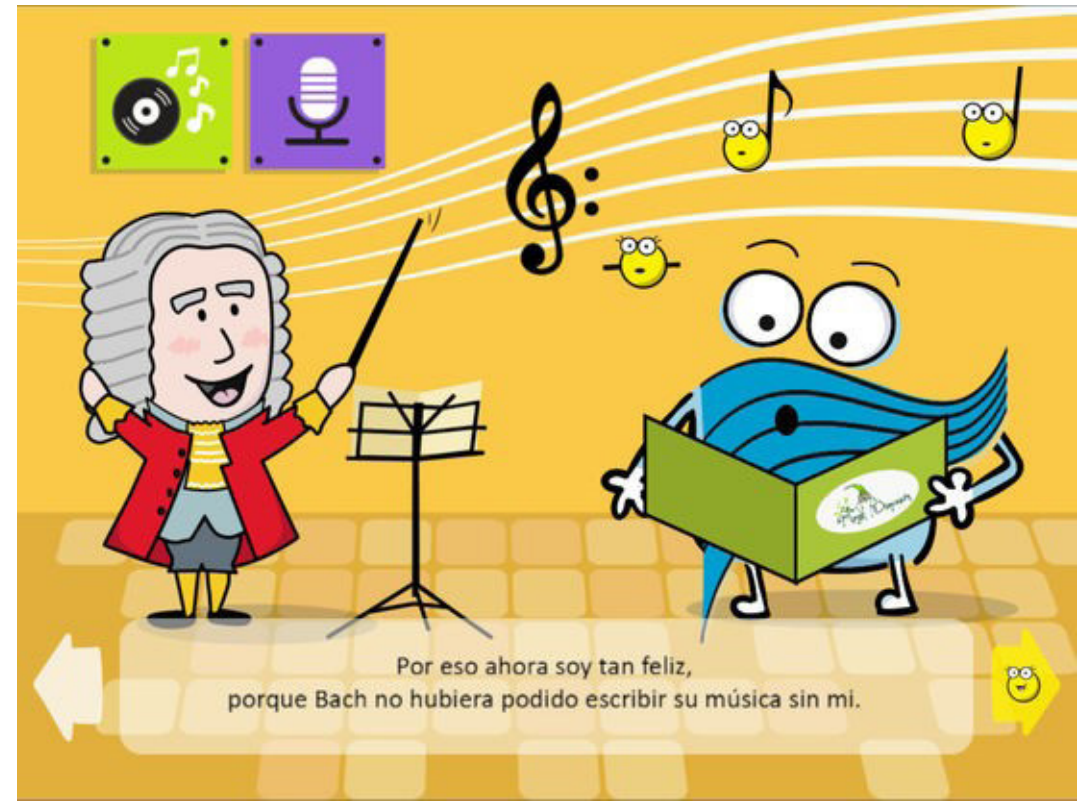

Figura 2. Ejemplo de una de las pantallas de la app "El Pentagrama"

\subsection{Objetivos de la investigación}

Teniendo en cuenta la escasez literaria sobre el desarrollo del oído absoluto en edades tempranas y considerando la metodología que ofrece el Mago Diapasón para desarrollar el oído absoluto en estas edades, esta investigación pretende examinar un método didáctico que contribuye al desarrollo del oído absoluto (OA) en niños de dos ańos, con la colaboración de los padres y madres, sin necesidad de tener conocimientos musicales. Para ello será imprescindible la ayuda de los recursos TIC propios de este Método. De este modo, el objetivo de esta investigación es examinar si los nińos de 2 ańos de edad, que siguen la metodología del Mago Diapasón y se apoyan de las apps propias de este método, empiezan a reconocer y afinar las notas La, Do, Mi y Sol. 
En definitiva, nos preguntamos si el uso de aplicaciones puede ayudar a cualquier niño a empezar a desarrollar el oído absoluto sin necesidad de tener familiares músicos. A partir de ello, las preguntas de investigación son:

- ¿ ¿Son los niños de dos años capaces de afinar las notas La, Do, Mi y Sol tras la implementación de la app del Mago Diapasón que ayuda a desarrollar el oído absoluto?

- ¿Existe diferencia de afinación entre aquellos niños que se han apoyado con la app del método de los que no lo han hecho?

\section{2.- Metodología}

\subsection{Participantes}

Los participantes del estudio han sido 19 alumnos de dos escuelas infantiles de un municipio de la provincia de Alicante. Un 42\% son nińas y un $58 \%$ son niños. De los 19 participantes, 9 de ellos conforman el grupo de control y los 10 restantes pertenecen al grupo experimental. Estos niños tienen una edad de dos ańos y el nivel socioeconómico de sus familias es medio. Todos los alumnos asisten a una clase de media hora semanal con la metodología "el Mago Diapasón”. Además, los participantes que conforman el grupo experimental trabajan con sus padres en casa el desarrollo del oído absoluto mediante la app (para Tablet y móvil) del Mago Diapasón. La selección del grupo de control se ha realizado de manera aleatoria de entre todos los alumnos de las dos escuelas infantiles y el grupo experimental se ha desarrollado con el consentimiento de los padres decididos a participar en este proyecto.

\subsection{Instrumentos}

Para llevar a cabo esta investigación se ha diseñado un cuestionario formado por 16 ítems agrupados en 3 bloques. Cada uno de los ítems consiste en un dictado musical de 7 notas. En cuanto a los bloques, estos siguen la estructura del Método Mago Diapasón en el que primero se trabaja la nota La, a continuación se introduce la nota Do, seguida de la nota Mi y finalmente la nota Sol, aumentando de este modo la dificultad a medida que se van introduciendo las notas. Así, el bloque 1 consiste en 6 ítems y el dictado musical contiene las notas La y Do. El bloque 2 está formado por 6 ítems y las notas usadas para el dictado son La, Do y Mi. Finalmente, el bloque 3 consiste en 4 ítems y el dictado musical está compuesto por las notas La, Do, Mi y Sol. A la hora de pasar el cuestionario se han tenido en cuenta dos variables: 
reconoce la nota y afina la nota. Para ello se puntuará entre 0 y 7 cada ítem según el número de aciertos tanto para la variable "reconoce la nota" como para la variable "afina la nota". La Figura 3 muestra el bloque 2 del cuestionario.

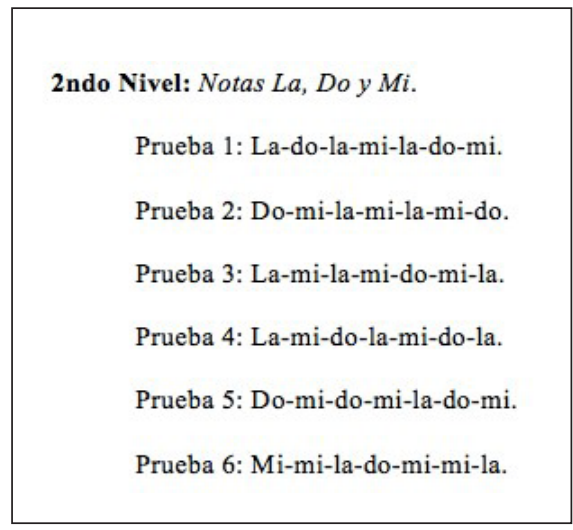

Figura 3. Bloque 2 del cuestionario

\subsection{Procedimiento}

En primer lugar, se pidió el consentimiento de los padres, tanto del grupo experimental como del grupo control, y se explicó a los padres del grupo experimental cómo se llevaría a cabo la investigación durante los seis meses que iba a durar esta. Este proceso de seis meses es fundamental, ya que el reconocimiento de los diferentes sonidos, alturas y aprender a cantar en estas edades es un proceso que requiere de un trabajo continuo y periódico.

Una vez explicada toda la información a los padres y a las escuelas infantiles, se pasó el cuestionario explicado en el apartado anterior (pre-test) a los 19 alumnos que conforman la muestra. Esto se llevó a cabo en octubre, cuando los alumnos llevaban solamente 3 clases del Mago Diapasón. Como ya se ha explicado, el test consistía en diferentes dictados musicales que eran interpretados por el profesor/ investigador, el cual anotaba si cada participante (de manera individual) reconocía las notas y si era capaz de afinarlas.

Durante seis meses, tanto el grupo experimental como el grupo control recibieron clases semanales de media hora siguiendo la metodología del Mago Diapasón (clases en grupo) y los participantes del grupo 
experimental, además, habían estado trabajando a través del juego con las aplicaciones del Mago Diapasón en casa. Al finalizar el período de aprendizaje de seis meses, se pasó de nuevo el mismo cuestionario (posttest) de manera individual a todos los participantes del estudio con el objetivo de examinar los cambios en el aprendizaje musical y el desarrollo del oído absoluto.

\subsection{Diseño y Análisis de datos}

Los datos del estudio fueron los resultados obtenidos tanto en el pre-test como en el post-test por parte de los 19 participantes. Para ello se tuvieron en cuenta dos variables: reconoce las notas y afina las notas, generándose tres categorías:

- No afina: el participante, o bien canta de manera errónea la nota que suena, o bien no contesta.

- Reconoce: el participante, o bien reconoce la nota que suena, pero no la afina, o bien la afina, pero nombra otra nota.

- Afina: el participante afina y reconoce la nota que suena.

Durante la realización del cuestionario el investigador anotaba si el participante no afinaba, reconocía o afinaba cada una de las notas de los dictados musicales. Seguidamente se obtuvo la puntuación total para cada una de las categorías a lo largo del cuestionario. Esta forma de puntuar se siguió, tanto para el cuestionario inicial (pre-test), como para el cuestionario final (post-test). Finalmente se calculó los porcentajes para cada una de las categorías. El motivo de unificar los datos en porcentajes es que algunos participantes no llegaron a resolver todo el cuestionario, ya que es difícil mantener su atención durante un periodo largo de tiempo a estas edades.

Tras examinar los datos obtenidos de forma individual para cada participante, se decidió confirmar los resultados a través de un análisis estadístico, a pesar de tener una muestra poco significativa (19 participantes). La evaluación del efecto del método para desarrollar el oído absoluto se llevó a cabo mediante un diseńo cuasi-experimental con grupo de control no equivalente y medias antes y después de la intervención en la categoría "afina". Los datos obtenidos siguiendo este diseño se analizaron mediante un análisis de la varianza factorial entre-sujetos e intra-sujetos, con la variable pretest-postest como factor de medidas repetidas y la variable tratamiento-no tratamiento como variable entre sujetos. Así, la 
variable independiente tiene dos niveles que son el grupo experimental y el grupo control, y la variable dependiente es el grado de desarrollo del oído absoluto, es decir, en qué medida los participantes han sido capaces o no de afinar las notas del cuestionario. El análisis estadístico se llevó a cabo con el programa estadístico SPSS Statistics.

\section{3.- Resultados}

A continuación, se presentan los resultados detallados en cuanto a la capacidad de afinar y reconocer las notas La, Do, Mi, Sol para el grupo experimental, el grupo de control, y los resultados estadísticos obtenidos con el programa SPSS.

\subsection{Resultados obtenidos del grupo experimental}

La Tabla 1 muestra los porcentajes de cada participante del grupo experimental para cada una de las categorías de análisis, tanto en el pre-test como en el post-test. Como ya se ha comentado en el análisis, los resultados aparecen en forma de porcentajes en lugar de datos absolutos para unificar los datos, ya que no todos respondieron el cuestionario completo.

Tabla 1. Porcentajes de cada participante del grupo experimental para cada una de las categorías

\begin{tabular}{|l|c|c|c|c|c|c|}
\hline \multicolumn{7}{|c|}{ GRUPO EXPERIMENTAL } \\
\hline \multirow{2}{*}{ PARTICIPANTE } & AFINA LA NOTA & \multicolumn{2}{c|}{ RECONOCE LA NOTA } & \multicolumn{2}{c|}{ NO AFINA LA NOTA } \\
\cline { 2 - 7 } & PRE-TEST & POST-TEST & PRE-TEST & POST-TEST & PRE-TEST & POST-TEST \\
\hline P01 & 0 & 28,6 & 23,8 & 51,4 & 76,2 & 20 \\
\hline P02 & 0 & 12,9 & 52,4 & 40,0 & 47,6 & 47,1 \\
\hline P03 & 0 & 38,6 & 38,1 & 17,1 & 61,6 & 44,3 \\
\hline P04 & 0 & 27,1 & 47,6 & 34,4 & 52,4 & 38,6 \\
\hline P05 & 0 & 14,3 & 0 & 21,4 & 0 & 64,3 \\
\hline P06 & 0 & 28,6 & 38,1 & 34,3 & 61,9 & 37,1 \\
\hline P07 & 0 & 25,0 & 42,9 & 16,7 & 57,1 & 58,3 \\
\hline P08 & 0 & 0 & 0 & 0 & 0 & 0 \\
\hline P09 & 0 & 0 & 33,3 & 0 & 66,7 & 0 \\
\hline P10 & 0 & 30,0 & 28,6 & 28,6 & 71,44 & 41,4 \\
\hline
\end{tabular}

En primer lugar cabe comentar que los participantes que tienen un $0 \%$ en el post-test (P08 y P09) se debe a que no han respondido al test. Este resultado no es calificativo, ya que el hecho de no tener respuesta no se ha podido evaluar o calificar. Cabe recordar que los participantes tienen dos ańos y en algunas ocasiones, quizá por vergüenza, no respondían al cuestionario, quedándose callados. 
Respecto a los resultados de este grupo para la categoría "afina la nota", se observa que en el pre-test ningún participante fue capaz de afinar ninguna nota. Este resultado era esperable, dado que el test se pasó al inicio del curso cuando los participantes todavía no tenían conocimientos musicales y, por tanto, no habían podido desarrollar la afinación. Sin embargo, se observa una mejora general en el post-test, ya que los participantes, después de seis meses recibiendo clases semanales y manipulando las apps en casa, empezaron a afinar entre el 12,9\% y el 30\% de las notas. En cuanto a las categorías "reconoce la nota" y "no afina la nota", que fueron las categorías que predominaron en el pre-test, podemos observar que en el post-test han disminuido los porcentajes. Esto es debido a que los participantes pasaron de no afinar o solamente reconocer la nota (en el pre-test), a empezar a afinar algunas notas en el post-test. Estos resultados sugieren que los participantes que, además de asistir a las clases semanales del Mago Diapasón, habían usado las apps, habían empezado a desarrollar el oído absoluto.

\subsection{Resultados obtenidos del grupo control}

La Tabla 2 muestra los porcentajes de cada participante del grupo control para cada una de las categorías de análisis, tanto en el pre-test como en el post-test.

Tabla 2. Porcentajes de cada participante del grupo control para cada una de las categorías

\begin{tabular}{|c|c|c|c|c|c|c|}
\hline \multicolumn{7}{|c|}{ GRUPO CONTROL $(\mathrm{N}=9)^{*}$} \\
\hline \multirow[t]{2}{*}{ PARTICIPANTE } & \multicolumn{2}{|c|}{ AFINA LA NOTA } & \multicolumn{2}{|c|}{ RECONOCE LA NOTA } & \multicolumn{2}{|c|}{ NO AFINA LA NOTA } \\
\hline & PRE-TEST & POST-TEST & PRE-TEST & POST-TEST & PRE-TEST & POST-TEST \\
\hline P11 & 0 & 8,6 & 0 & 35,7 & 0 & 55,7 \\
\hline P12 & 0 & 7,1 & 0 & 21,4 & 0 & 71,4 \\
\hline P13 & 0 & 0 & 0 & 0 & 0 & 0 \\
\hline P14 & 0 & 0 & 0 & 0 & 0 & 0 \\
\hline P15 & 0 & 0 & 0 & 0 & 0 & 0 \\
\hline P16 & 0 & 0 & 71,4 & 0 & 28,6 & 0 \\
\hline P17 & 0 & 0 & 0 & 0 & 0 & 0 \\
\hline P18 & 0 & 7,1 & 0 & 31,4 & 0 & 61,4 \\
\hline P19 & 0 & 0 & 0 & 0 & 0 & 0 \\
\hline
\end{tabular}

En primer lugar, cabe comentar que, a excepción de P16, los participantes del grupo control no respondieron al cuestionario inicial (pre-test), obteniendo un $0 \%$ en todas las categorías.

En cuanto al post-test, la mayoría de los participantes continuaron sin responder el cuestionario. Solamente P11, P12 y P18 lo respondieron. Respecto a los resultados de estos tres participantes para la 
categoría "afina la nota", se observa que sí fueron capaces de afinar algunas notas (8,6\%, 7,1\% y 7,1\%, respectivamente), aunque predominan las otras dos categorías. A pesar de que ha habido una mejora a la hora de afinar algunas notas, si lo contrastamos con el grupo experimental podemos observar que la mejora ha sido mucho más significativa en el primer grupo. Este resultado sugiere que las clases del Mago Diapasón ayudan a empezar a desarrollar el oído absoluto. Sin embargo, esta metodología resulta más efectiva si se complementa con el uso de las apps correspondientes.

\subsection{Resultados estadísticos obtenidos para la variable "afina las notas"}

Como ya se ha comentado en la sección de análisis, y siendo conscientes del tamaño poco significativo de la muestra, para corroborar los resultados observados a través de los porcentajes de cada participante del estudio y confirmar si influye la práctica a partir de los recursos TIC para ayudar a desarrollar el oído absoluto, se llevó a cabo un diseńo cuasi-experimental con grupo control no equivalente y medias antes y después de la intervención. Para el análisis factorial realizado, se consideró la variable momento (pretest y post-test, intra sujetos) y la variable condición experimental (grupo experimental y grupo control, entre sujetos). La evaluación del Método Mago Diapasón a partir de sus apps se realizó para el grupo experimental.

Los resultados descriptivos para los grupos experimental y control se presentan de forma esquemática en la Tabla 3. Se observa que la media varía en el grupo experimental antes y después, obteniendo unas medias de 0,00 y 20,51, respectivamente. Por otro lado, el grupo control también varía las medias $(0,00$ antes y 2,53 después), pero no de manera tan significativa como el grupo experimental. Este resultado sugiere que las clases semanales del Mago Diapasón han ayudado a los participantes a afinar algunas notas, pero el uso de las aplicaciones con la ayuda de los padres ha ayudado a afinar las notas significativamente.

En la Tabla 3 se presentan de forma esquemática los resultados relacionados con el análisis factorial de la varianza con medias repetidas en la variable afina las notas. Como podemos observar, se produce un efecto claramente significativo, tanto en la variable entre-sujetos, intra-sujetos, como en la interacción. Respecto a la variable intra-sujetos, la puntuación de ambos grupos tomados en conjunto cambia significativamente desde el inicio del estudio hasta la finalización del mismo $(F(1,19)=25,656, p=.000)$. Respecto a la variable entre-grupos, también se observan diferencias entre el grupo control y grupo experimental $(F(1,19)=$ $15,614, \mathrm{p}=.000)$. Para establecer el posible efecto del uso de las aplicaciones para ayudar a desarrollar el 
oído absoluto, se examina la interacción entre las variables grupo x momento de evaluación del Método, y se obtienen valores significativos para la variable "afina las notas" $(\mathrm{F}(1,19)=15,614, \mathrm{p}=.001)$, es decir, como la interacción resulta significativa, se produce un cambio diferencial en uno y otro grupo, tal y como se observa en la Tabla 4 y en la Figura 4. Este análisis muestra que la práctica a partir de los recursos TIC influye para ayudar a desarrollar el oído absoluto.

Tabla 3. Resultados descriptivos sobre las puntuaciones de la categoría afina las notas

\begin{tabular}{|l|l|c|c|c|}
\hline \multicolumn{2}{|c|}{ AFINA LAS NOTAS } & $\mathrm{X}$ & $\mathrm{DT}$ & $\mathrm{N}$ \\
\hline \multirow{4}{*}{ ANTES } & CONTROL & 0,000 & 0,0000 & 9 \\
\cline { 2 - 5 } & EXPERIMENTAL & 0,000 & 0,0000 & 10 \\
\cline { 2 - 5 } & TOTAL & 0,000 & 0,0000 & 19 \\
\hline \multirow{3}{*}{ DESPUÉS } & CONTROL & 2,533 & 3,8246 & 9 \\
\cline { 2 - 5 } & EXPERIMENTAL & 20,510 & 13,1216 & 10 \\
\cline { 2 - 5 } & TOTAL & 11,995 & 13,3279 & 19 \\
\hline
\end{tabular}

Tabla 4. Resultados del análisis factorial de la varianza realizado sobre las puntaciones de la categoría afina las notas

\begin{tabular}{|c|c|c|}
\hline EFECTO & $\mathrm{F}$ & $\mathrm{P}$ \\
\hline ENTRE SUJETOS & 15,614 &, 001 \\
\hline INTRA SUJETOS & 25,656 &, 000 \\
\hline INTERACCIÓN & 15,614 &, 001 \\
\hline
\end{tabular}

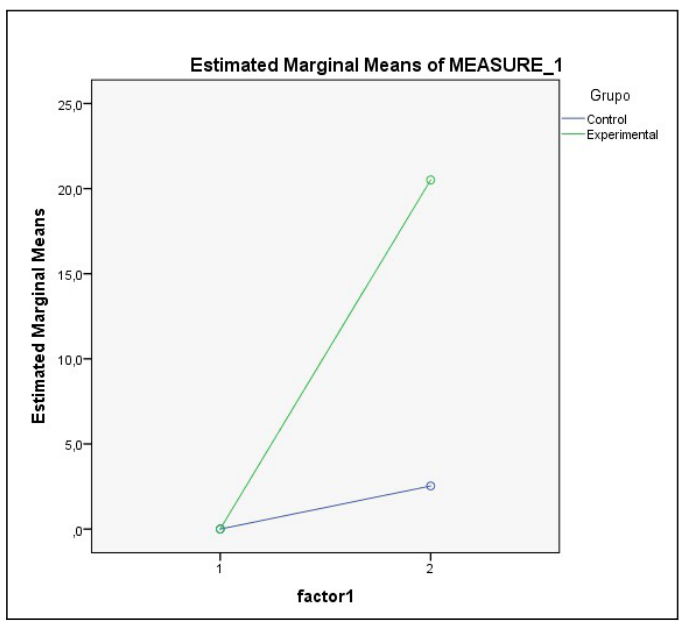

Figura 4. Gráfica de la intersección entre el grupo (experimental y control) y la medida pre y post tratamiento para la categoría "afina las notas"

En la Figura 4 se pueden apreciar las diferencias de medias entre el grupo experimental y el grupo control 
para la variable "afina las nota", antes y después del tratamiento. Como vemos, se observa que, al inicio del curso, cuando se pasó el cuestionario inicial, los participantes no fueron capaces de afinar ninguna nota, y tras 6 meses con las clases semanales del Mago Diapasón, ambos grupos (control y experimental) han empezado a afinar algunas notas. Sin embargo, los participantes del grupo experimental que son los que, además de las clases, han trabajado con las apps durante este periodo de tiempo, han obtenido resultados mucho más significativos a la hora de afinar las notas.

\section{4.- Discusión y conclusiones}

Las preguntas de investigación de este estudio eran, por una parte, examinar si los niños de dos años son capaces de afinar las notas, La, Do, Mi y Sol, tras la implementación de un programa que ayuda a desarrollar el oído absoluto y, por otra parte, si existe diferencia entre aquellos niños que han asistido a las clases y utilizado las apps (específicas Mago Diapasón), de aquellos que solamente han asistido a las clases. Los resultados, tras analizar el pre-test y post-test, tanto en el grupo experimental, como en el de control, nos llevan a concluir una serie de aspectos referidos a las preguntas de investigación.

En primer lugar, respecto a la primera pregunta de investigación, es decir, analizar si con la implementación de un programa sobre oído absoluto los niños de dos años pueden afinar las notas La, Do, Mi y Sol, cabe decir que en el pre-test (inicio del curso), algunos participantes fueron capaces de reconocer algunas de ellas (en particular La y Do), dado que tenían ya algunas clases previas con la metodología del Mago Diapasón. Sin embargo, otros participantes no respondieron a las preguntas del cuestionario. Esto podría deberse a que los niños, con solo dos años, o bien no entendieron que cuando se exponen a un test, tienen que responder a las preguntas, o bien al carácter del niño a la hora de responder (algunos se mostraban tímidos, otros respondían en voz muy bajita (solo gesticulando con los labios), y otros se bloqueaban y no contestaban). En cuanto a los resultados del pre-test a la hora de afinar, no hubo ningún participante que fuese capaz de afinar alguna nota. Este resultado era esperado, dado que los niños tenían 2 ańos y previamente al test no tenían apenas ningún conocimiento musical.

Los resultados del post-test muestran que, después del periodo de enseñanza-aprendizaje del Mago Diapasón, los participantes del estudio han mejorado a la hora de reconocer y entonar las notas La, Do, Mi y Sol (presentadas progresivamente en este riguroso orden), tanto en el grupo de control como en el experimental. Este resultado sugiere que aprender música con esta metodología ayuda a los niños a 
introducirse en el lenguaje musical y a desarrollar el oído absoluto.

Respecto a la segunda pregunta de investigación, es decir, analizar la diferencia en el desarrollo del oído absoluto entre niños al utilizar o no una app específica sobre música, cabe decir que, aun sabiendo que las clases introducen al lenguaje musical y ayudan a desarrollar el oído absoluto, el uso de las apps específicas que ofrece el Mago Diapasón para sus estudiantes, incentivan y aceleran el proceso para desarrollar el oído de manera más efectiva. Este resultado se observa al comparar las medias del grupo experimental y grupo control, ya que los participantes del grupo experimental afinan entre un $12,9 \%$ y $30 \%$ de las notas, tras seis meses de jugar con las apps. Sin embargo, los participantes del grupo control afinan entre un $7 \%$ y un $8 \%$ de las notas (teniendo en cuenta solamente los participantes del grupo de control que respondieron al cuestionario). Este resultado sugiere que el uso de esta app permite a los nińos ampliar sus conocimientos musicales.

Según Baharloo et al. (1998), los hermanos menores que escuchan cómo sus hermanos mayores estudian música (tocan algún instrumento), tienen muchas más probabilidades de desarrollar el OA. Esta idea está en línea con nuestro trabajo, donde la tecnología actúa de emisor. Por otro lado, el uso de las apps puede sustituir en su defecto a los instrumentos de tono fijo y es que, coincidiendo con Vanzella y Schellenberg (2010), la escucha del piano u otros instrumentos de tono fijo parecen mejorar las habilidades del OA y extender el período sensible para la exposición a la música con el fin de desarrollar un OA más preciso.

Finalmente, los resultados indican que afinar las notas ( $\mathrm{La}$, Do, Mi y Sol) es más difícil que el simple reconocimiento de las mismas cuando se escuchan. Esto se ve evidenciado en los porcentajes, tanto del grupo experimental como de control, en el pre-test y en el post-test. En concreto, en el pre-test ningún participante afina ninguna nota. Sin embargo, a pesar de llevar solamente 3 clases del Mago Diapasón, sí son capaces de reconocer parte de ellas. En el post-test, también observamos que los porcentajes son más elevados en la categoría de reconocer las notas que en la categoría de afinar las notas. Este resultado sugiere que reconocer las notas musicales es un paso previo para poder afinarlas. Como limitación al estudio, cabe decir que, tanto la muestra como el periodo de aprendizaje, han sido pequeńos.

\section{Financiación}

El presente trabajo se enmarca en el seno del Grupo de Investigación "GIDU-EDUTIC-IN", del Pro- 
grama de Redes de Investigación en Docencia Universitaria del ICE (REDES-I3CE-2018-4423) y del proyecto Instituto Superior de Investigación Cooperativa IVITRA (Ref.: ISIC/2012/022; http://www. ivitra.ua.es), todos ellos de la Universidad de Alicante; y del proyecto "Second Round Art i Lluita a Secundària en Cinema i Audiovisuals" (UV-SFPIE_GER18-848892) de la Universitat de València.

\section{Referencias}

Arlandis, S., \& Reyes-Torres, A. (2018). Thresholds of Change in Children's Literature: The Symbol of the Mirror. Journal of New Approaches in Educational Research, 7(2), 125-130. http://dx.doi. org/10.7821/naer.2018.7.275

Baharloo, S., Johnston, P. A., Gitschier, J., \& Freimer, N. B. (1998). Absolute pitch: an approach for identification of genetic and nongenetic components. The American Journal of Human Genetics, 62(2), 224-231. https://doi.org/10.1086/301704

Baharloo, S., Risch, N., Gitschier, J., \& Freimer, N. B. (2000). Familial aggregation of absolute pitch. The American Journal of Human Genetics, 67(3), 755-758. https://doi.org/10.1086/303057

Barkowsky, J. J. (1987). An investigation into pitch identification behavior of absolute pitch and relative pitch subjects. Doctoral dissertation, University of Illinois at Urbana-Champaign.

Cabero-Almenara, J. \& Roig-Vila, R. (2019). The Motivation of Technological Scenarios in Augmented Reality (AR): Results of Different Experiments. Applied Sciences, 9(14). https://doi.org/10.3390/ app9142907

Claro, M. (2010). Documento de Proyecto: Impacto de las TIC en los aprendizajes de los estudiantes. Estado del arte. Recuperado en: http://goo.gl/3Cvfp4

Creer, A. (2018). Introducing Everyday Digital Literacy Practices into the Classroom: An Analysis of Multi-layered Media, Modes and their Affordances. Journal of New Approaches in Educational Research, 7(2), 131-139. http://dx.doi.org/10.7821/naer.2018.7.265

Dohn, A., Garza-Villarreal, E.A., Heaton, P., \& Vuust, P. (2012). Do Musicians with Perfect Pitch Have More Autism Traits than Musicians without Perfect Pitch? An Empirical Study. PloS One 7(5): e37961.

Froehlich, D. (2018). Non-Technological Learning Environments in a Technological World: Flipping Comes To The Aid. Journal of New Approaches in Educational Research, 7(2), 88-92. doi: http://dx.doi. 
$\operatorname{org} / 10.7821 /$ naer.2018.7.304

García-Umaña, A., \& Tirado-Morueta, R. (2018). Digital Media Behavior of School Students: Abusive Use of the Internet. Journal of New Approaches in Educational Research, 7(2), 140-147. doi: http:// dx.doi.org/10.7821/naer.2018.7.284

Heaton, P. (2009). Assessing musical skills in autistic children who are not savants. Philosophical Transactions of the Royal Society B. Biological Sciences, 364(1522), 1443-1447. https://doi. org/10.1098/rstb.2008.0327

Hincapié, V. M., \& Graciano, M. (2016). La música en los procesos de lectoescritura para la educación inicial a partir de las TICS y las artes visuales. Bachelor's thesis, Escuela de Educación y Pedagogía.

Lánchez, M. (2014). TICS, lectura y música: una aproximación al diseño instruccional desde el enfoque de la neuroeducación. TFM: UNED, Facultad de educación.

Llargués, M., Niño, S., Rodríguez, N., \& Segura, E. (2015). Tic y clases de música. En M. Ramos, A. Reynaldi, L. Pinto, y A. Fulgueiras, Primera Jornada de teoría y práctica de la enseñanza musical (pp. 43-52). Universidad Nacional de la Plata.

Marín-Díaz, V., Morales-Díaz, M., \& Reche-Urbano, E. (2019). Educational Possibilities of Video Games in the Primary Education Stage According to Teachers in Training. A Case Study. Journal of New Approaches in Educational Research, 8(1), 42-49. http://dx.doi.org/10.7821/naer.2019.1.330

Martino G., \& Frida M. (2014). Las tecnologías de la información y comunicación y el bienestar psicológico en la generación net. Revista Hamut'ay, 1(1). Recuperado en: https://bit.ly/2pI3q11

Miyazaki, K. (1988). Musical pitch identification by absolute pitch possessors. Perception \& Psychophysics, $44(6), 501-512$.

Miyazaki, K. (1994). Recognition of transposed melodies by absolute pitch listeners. En Proceeding of the 3rd International Conference for Music Perception and Cognition Proceedings (pp. 295-296). Liège : Irène Deliège, editor. Université de Liège.

Miyazaki, K. (1995). Perception of relative pitch with different references: Some absolute-pitch listeners can't tell musical interval names. Perception \& Psychophysics, 57(7), 962-970.

Morel, T. T. (2016). Música y tecnología: taller para la integración de las TIC en el aula de educación musical. Contextos: Estudios de Humanidades y Ciencias Sociales, 27, 109-124.

Moulton, C. (2014). Perfect pitch reconsidered. Clinical Medicine, 14(5), 517-519. 
Nering, M. E. (1991). A study to determine the effectiveness of the David L. Burge technique for development of perfect pitch. Michigan: UMI Dissertation Services

Pastor, A. (2008). Matemáticas en la música. SUMA 59, 17-21.

Radocy, R. E., \& Boyle, J. D. (1988). Affective behaviors and music Affective behaviors and music. Psychological foundations of musical behavior, 14(5), 195-242.

Reinking, A., \& Martin, B. (2018). The Gender Gap in STEM Fields: Theories, Movements, and Ideas to Engage Girls in STEM. Journal of New Approaches in Educational Research, 7(2), 148-153. doi:http:// dx.doi.org/10.7821/naer.2018.7.271

Roig-Vila, R., Lorenzo-Lledó, A., \& Mengual-Andrés, S. (2019). Utilidad percibida de la realidad aumentada como recurso didáctico en Educación Infantil. Campus Virtuales, 8(1), 19-35. Recuperado de: https://bit.ly/2nVDyO0

Sánchez, J. (2003). Integración Curricular de TICs. Concepto y Modelos. Revista Enfoques Educacionales, 5(1), 51-65.

Shuter-Dyson, R. \& Gabriel, C. (1981). The Psychology of Musical Ability. London: Methuen.

Stumpf, C. (1883). Tonpsychologie. Leipzig: Hirzel.

Vanzella, P. \& Schellenberg, E. G. (2010). Absolute pitch: Effects of timbre on note-naming ability. PloS one, 5(11), e15449.

Zielinska, H., \& Miklaszewski, K. (1992). Memorising two melodies of different style. Psychology of Music, 20(2), 95-111.

The Design-Based Research Collective (2003). Design-Based Research: An Emerging Paradigm for Educational Inquiry. EducationalResearcher, 32(1), 5-8.https://doi.org/10.3102/0013189X032001005

\section{Cómo citar este artículo:}

Roig-Vila, R., San Blas, F. \& Buforn, A. (2020). Desarrollo del oído absoluto a través de aplicaciones móviles. Pixel-Bit. Revista de Medios y Educación, 57, 191-209. https://doi.org/10.12795/ pixelbit.2020.i57.08 